\title{
ON THE STRUCTURE OF UNITARY GROUPS
}

\author{
BY \\ JEAN DIEUDONNE
}

1. Let $K$ be an arbitrary sfield with an involution $J$, that is, a one-to-one mapping $\xi \rightarrow \xi^{J}$ of $K$ onto itself, distinct from the identity, such that $(\xi+\eta)^{J}$ $=\xi^{J}+\eta^{J},(\xi \eta)^{J}=\eta^{J} \xi^{J}$, and $\left(\xi^{J}\right)^{J}=\xi$. Let $E$ be an $n$-dimensional right vector space over $K(n \geqq 2)$; an hermitian (resp. skew-hermitian) form over $E$ is a mapping $(x, y) \rightarrow f(x, y)$ of $E \times E$ into $K$ which, for any $x$, is linear in $y$, and such that $f(y, x)=(f(x, y))^{J}$ (resp. $\left.f(y, x)=-(f(x, y))^{J}\right)$. This implies that $f(x, y)$ is additive in $x$ and such that $f(x \lambda, y)=\lambda^{J} f(x, y)$. The values $f(x, x)$ are always symmetric (resp. skew-symmetric) elements of $K$, that is, elements $\alpha$ such that $\alpha^{J}=\alpha$ (resp. $\left.\alpha^{J}=-\alpha\right)$. The orthogonality relation $f(x, y)=0$ relative to $f$ is always symmetric.

We shall always suppose that the form $f$ is nondegenerate, or in other words that there is no vector in $E$ other than 0 orthogonal to the whole space. Moreover, when the characteristic of $K$ is 2, the distinction between hermitian and skew-hermitian forms disappears, and $f(x, x)$ is symmetric for every $x \in E$; in that case we shall make the additional assumption that $f(x, x)$ has always the form $\xi+\xi^{J}$ ("trace" of $\xi$ ) for a convenient $\xi \in K$; this assumption is automatically verified when the restriction of $J$ to the center $Z$ of $K$ is not the identity, but not necessarily in the other cases.

A unitary transformation $u$ of $E$ is a one-to-one linear mapping of $E$ onto itself such that $f(u(x), u(y))=f(x, y)$ identically; these transformations constitute the unitary group $U_{n}(K, f)$. In a previous paper [5, pp. 63-82]( $\left.{ }^{1}\right)$, I have studied the structure of that group in the two simplest cases, namely those in which $K$ is commutative, or $K$ is a reflexive sfield and the form $f$ is hermitian; the present paper is devoted to the study of $U_{n}(K, f)$ in the general case.

2. We shall need the following lemma:

Lemma 1. If the sfield $K$ is not commutative, it is generated by the set $S$ of the symmetric elements, except when $K$ is a reflexive sfield of characteristic $\neq 2$, and $S$ is identical with the center $Z$ of $K$.

Let $L$ be the subsfield of $K$ generated by $S$; we are going to prove that if $L$ is not contained in $Z$, then $L=K$. Suppose the contrary, and let $\alpha$ be an element in $K$ not belonging to $L$; let $M$ be the 2-dimensional right vector space over $L$ having 1 and $\alpha$ as a basis; we are going to prove that $M$ is a sfield. We first notice that $L$ is identical with the subring of $K$ generated by $S$;

Received by the editors August 14, 1951.

(1) Numbers in square brackets refer to the bibliography at the end of the paper. 
for if $\zeta \neq 0$ is an element of that subring, it is clear that $\zeta^{J}$ also belongs to it; but $\zeta \zeta^{J}=\delta$ is in $S$, hence $\zeta^{-1}=\zeta^{J} \delta^{-1}$ belongs to the ring generated by $S$, which proves that $L$ is identical with that ring. We next notice that $\alpha^{J}+\alpha=\beta$ $\in S \subset L$, and $\alpha \alpha^{J}=-\alpha^{2}+\alpha \beta=\gamma \in L$ and therefore $\alpha^{2}=\alpha \beta-\gamma$. On the other hand, if $\xi$ is any element in $S, \alpha \xi+(\alpha \xi)^{J}=\alpha \xi+\xi \alpha^{J}$ is in $L$, and therefore $\alpha \xi-\xi \alpha$ is in $L$; by induction on $k$, it follows that if $\zeta=\xi_{1} \xi_{2} \cdots \xi_{k}$, where $\xi_{j} \in S$ for $1 \leqq j \leqq k$, the element $\alpha \zeta-\zeta \alpha$ is in $L$. These remarks prove that $M$ is a subring of $K$, invariant by the involution $J$, and the same argument as the one made for $L$ proves that $M$ is a sfield. Now for any $\zeta \in L, \alpha \zeta+(\alpha \zeta)^{J}=\alpha \zeta+\zeta^{J} \alpha^{J}$ is in $S \subset L$, and replacing $\alpha^{J}$ by its value shows that $\alpha \zeta-\zeta^{J} \alpha$ is in $L$; but as $\zeta^{J} \alpha-\alpha \zeta^{J}$ also belongs to $L$, we see that $\alpha\left(\zeta-\zeta^{J}\right)$ is in $L$; this is of course possible only when $\zeta=\zeta^{J}$. In other words, we come to the conclusion that $L=S$; in particular, if $\xi$ and $\eta$ are any two elements of $S, \xi \eta$ is in $S$, and therefore $(\xi \eta)^{J}=\eta^{J} \xi^{J}$ $=\eta \xi$ is equal to $\xi \eta$; this means that $L$ is a commutative field.

To go on with the argument, let us first suppose that the characteristic of $K$ is $\neq 2$; then, as $\alpha=\left(\alpha+\alpha^{J}\right) / 2+\left(\alpha-\alpha^{J}\right) / 2, \alpha-\alpha^{J}$ is not in $L$, and we can replace $\alpha$ by $\alpha-\alpha^{J}$ in the preceding sequence of arguments. We then have $\alpha^{J}=-\alpha$, and $\alpha^{2}=-\gamma \in L$. The mapping $\zeta \rightarrow \alpha \zeta-\zeta \alpha$ is a derivation of the field $L$; if we put $D \zeta=\alpha \zeta-\zeta \alpha$, we have $D^{2} \zeta=\alpha^{2} \zeta-2 \alpha \zeta \alpha+\zeta \alpha^{2} \in L$ for every $\zeta \in L$, which gives $\alpha \zeta \alpha \in L$, since the characteristic of $L$ is $\neq 2$. But we may write $\alpha \zeta \alpha=\alpha^{2} \zeta-\alpha \cdot D \zeta$ and as $\alpha^{2} \in L$, this gives $\alpha \cdot D \zeta \in L$, which is possible only if $D \zeta=0$ for every $\zeta \in L$. This proves that every element $\alpha \in K$ commutes with every element of $L$, in other words, that $L$ is in the center of $K$, contrary to assumption.

We next take up the case in which the characteristic of $K$ is 2. From the relation $\alpha^{3}=\alpha \beta \alpha-\gamma \alpha=\alpha^{2} \beta-\alpha \gamma$, one derives immediately $D \beta=D \gamma=0$, in other words, $\beta$ and $\gamma$ commute with $\alpha$; replacing $\alpha$ by $\beta^{-1} \alpha$, we can therefore suppose that $\alpha^{2}=\alpha+\gamma$, with $D \gamma=0$. Let $N$ be the subfield of $L$ defined by the equation $D \xi=0$ (commuting subfield of $\alpha$ or center of $M$ ). The relation $\alpha^{2}=\alpha+\gamma$ implies that $D^{2} \xi=D \xi$ for every $\xi \in L$, or in other words, that $\xi+D \xi \in N$ for all $\xi \in L$. On the other hand, $D\left(\xi^{2}\right)=2 \xi \cdot D \xi=0$ because the characteristic is 2 , hence $\xi^{2} \in N$ for $\xi \in L$. Now, if $\zeta=\alpha \xi+\eta$ is any element of $M$, with $\xi \in L$ and $\eta \in L$, an easy computation shows that $\zeta \zeta^{J}=\gamma \xi^{2}+\xi \eta$ $+D(\xi \eta)+\eta^{2}$ and therefore $\zeta \zeta^{J} \in N$; on the other hand $\zeta+\zeta^{J}=\xi+D \xi$ is also in $N$. If $N \neq L$, this means that $M$ is a reflexive sfield over its center $N[5, \mathrm{p}$. $72]$. But in a reflexive sfield of characteristic 2, the symmetric elements constitute a 3-dimensional subspace over the center, whilst here they are the elements of $L$, which is only 2-dimensional over $N$; the assumption $N \neq L$ is therefore untenable. But if $N=L, \alpha$ commutes again with every element of $L$, in other words, $L$ is again the center of $K$, contrary to assumption.

We have still to examine the exceptional case in which $S$ is contained in $Z$. For every element $\xi \in K, \xi+\xi^{J}$ and $\xi^{J} \xi$ are then in the center $Z$, and therefore, as $\xi^{2}-\left(\xi+\xi^{J}\right) \xi+\xi^{J} \xi=0$, every element of $K$ has degree 2 over the center 
$Z$. It is well known that this is possible only if $K$ has rank 4 over $Z$. Moreover if $\gamma \in Z$ and $\zeta$ is not in $Z, \gamma \zeta+(\gamma \zeta)^{J}=\gamma\left(\zeta+\zeta^{J}\right)+\left(\gamma^{J}-\gamma\right) \zeta^{J}$ is in $Z$, which implies $\gamma^{J}=\gamma$; this shows that $K$ is a reflexive sfield [5, p. 72], and $S=Z$; but this is possible only when $K$ has a characteristic $\neq 2$ (loc. cit.), and that completes the proof of Lemma 1 .

3. From the involution $J$, we can deduce other involutions $T$ of $K$ by the general process of setting $\xi^{T}=p^{-1} \xi^{J} p$, where $p$ is a symmetric or skew-symmetric element of $K$ (with respect to $J)$; if $p^{J}=\epsilon p(\epsilon=1$ or $\epsilon=-1)$, the relation $\xi^{T}=\xi$ is then equivalent to $p \xi=\epsilon(p \xi)^{J}$; in other words, the $T$-symmetric elements of $K$ are of the form $p^{-1} \eta$, where $\eta$ is $J$-symmetric if $\epsilon=1$ and $\eta$ is $J$-skew-symmetric if $\epsilon=-1$. This enables one to reduce to each other the hermitian and skew-hermitian forms, by a change of the involution (when the characteristic of $K$ is not 2). Indeed, if $f(y, x)=-(f(x, y))^{J}$, consider the form $g(x, y)=p^{-1} f(x, y)$, where $p$ is skew-symmetric; then $g$ is linear in $y$, and one has $g(y, x)=-p^{-1}(f(x, y))^{J}=-p^{-1}(p g(x, y))^{J}=(g(x, y))^{T}$. For the sake of convenience, we shall always suppose in the following that the form $f$ is skew-hermitian for $J$.

The notions of orthogonal basis, of isotropic vector, of isotropic and totally isotropic subspaces of $E$ are defined as usual (see [5]); the index $\nu$ of $f$ is the maximum dimension of the totally isotropic subspaces, and one has $2 \nu \leqq n$. When a plane $P \subset E$ is not totally isotropic but contains an isotropic vector $a \neq 0$, then there exists in $P$ a second isotropic vector $b$ such that $f(a, b)=1$; $P$ is then said to be a hyperbolic plane, and the restrictions of $f$ to any two hyperbolic planes are equivalent. Moreover, Witt's theorem is still valid (see [6, pp. 8-9]; in the case of characteristic 2, this, as well as the preceding property, is due to the restrictive assumption on $f$ to be "trace-valued"); we shall formulate it in the following form: if $V$ and $W$ are any two subspaces of $E$ such that the restrictions of $f$ to $V$ and $W$ are equivalent, then there is a unitary transformation $u$ such that $u(V)=W$.

4. Let us recall that a transvection is a linear transformation of the type $x \rightarrow x+a \rho(x)$, where $\rho$ is a linear form, not identically 0 , and such that $\rho(a)=0$. If we write that such a transformation is unitary, we get

$$
(\rho(x))^{J} f(a, y)+f(x, a) \rho(y)+(\rho(x))^{J} f(a, a) \rho(y)=0
$$

identically in $x$ and $y$; with $x=a$ this gives $f(a, a) \rho(y)=0$, hence $f(a, a)=0$, the vector $a$ must be isotropic; then we get

$$
(\rho(x))^{J} f(a, y)+f(x, a) \rho(y)=0
$$

which, for fixed $x$ such that $\rho(x) \neq 0$, shows that $f(x, a) \neq 0$, and $\rho(y)=\lambda f(a, y)$; finally, we have

$$
(f(a, x))^{J} \lambda^{J} f(a, y)+f(x, a) \lambda f(a, y)=0
$$

identically, and as $f(a, x)=-(f(x, a))^{J}$, this yields $\lambda^{J}=\lambda$. In other words, 
unitary transvections exist only if $\nu \geqq 1$, and then are of the form $x \rightarrow x$ $+a \lambda f(a, x)$, where $a$ is an arbitrary isotropic vector, and $\lambda$ an arbitrary symmetric element in $K$; the hyperplane of points of $E$ invariant by the transvection is the hyperplane orthogonal to $a$.

Let $H$ be a nonisotropic hyperplane, $a$ a vector orthogonal to $H$. Then every unitary transformation $u$ leaving invariant every element of $H$ is such that $u(a)=a \mu$, with $\mu^{J} \alpha \mu=\alpha$, where $\alpha=f(a, a)$; we shall say that such a transformation is a quasi-symmetry. There always exist quasi-symmetries of hyperplane $H$, not reduced to the identity; this is obvious if $K$ has a characteristic $\neq 2$, for then the ordinary symmetry $(\mu=-1)$ has that property. If $K$ has characteristic 2 , one has by assumption $\alpha=\beta+\beta^{J}$, with $\beta \neq \beta^{J}$; then $\mu=\beta^{-1} \beta^{J}$ satisfies $\mu^{J} \alpha \mu=\alpha$, and $\mu \neq 1$.

These remarks already enable us to determine the center $Z_{n}$ of the group $U_{n}(K, f)$. Indeed, a transformation $v$ belonging to the center must permute with every quasi-symmetry, hence leave invariant every nonisotropic line; and if there are isotropic lines, $v$ must permute with every unitary transvection, hence leave invariant every isotropic line as well. Therefore $v$ leaves invariant every line, which means that it is a homothetic mapping $x \rightarrow x \gamma$, with $\gamma$ in the center $Z$ of $K$ and $\neq 0$; moreover, in order that such a mapping be unitary, it is necessary and sufficient that $\gamma^{J} \gamma=1$.

5. From now on, we are going to suppose that $\nu \geqq 1$. Let $T_{n}$ be the subgroup of $U_{n}(K, f)$ generated by unitary transvections; as a transform $v u v^{-1}$ of a transvection $u$ is again a transvection, it is clear that $T_{n}$ is a normal subgroup of $U_{n}$. Let $W_{n}$ be the center of $T_{n}$ (we shall determine its structure in $\S 11$ ). We shall now prove the following theorem.

THEOREM 1. If the sfield $K$ has more than 25 elements $\left({ }^{2}\right)$, the group $T_{n} / W_{n}$ is simple for $n \geqq 2$ and $\nu \geqq 1$.

Our proof will be modeled after that of [5, Theorem 4, p. 55], and will proceed in several steps.

$1^{\circ}$. We first prove that if a normal subgroup $G$ of $T_{n}$ contains all transvections of $U_{n}$ having the same vector $a$, then $G=T_{n}$. In order to do this, we shall prove the following lemma.

LEMMA 2, If $a$ and $b$ are any two noncollinear isotropic vectors, there exists a transformation $u \in T_{n}$ such that $u(a)=b \mu$ for a convenient scalar $\mu \in K$.

If we suppose the lemma proved, and consider an arbitrary transvection $x \rightarrow v(x)=x+a \alpha f(a, x)$, it is readily verified that $u v u^{-1}$ is the transvection $x \rightarrow x+b \mu \alpha \mu^{J} f(b, x)$; but as $\alpha$ can take any value in the set $S$ of symmetric elements, so can $\mu \alpha \mu^{J}$. Therefore $G$ contains all transvections of $b$, and in consequence is identical to $T_{n}$, since $b$ is an arbitrary isotropic vector.

(2) The theorem is still true when $K$ has at most 25 elements, except when $K=F_{4}, n=2$ and $n=3$, and $K=F_{9}, n=2[5$, p. 70$]$. 
To prove the lemma, let us first suppose that $f(a, b) \neq 0$; then there is a scalar $\mu \neq 0$ such that $a+b \mu=c$ is isotropic. Indeed, the relation $f(a+b \mu, a+b \mu)=0$ gives the condition $\mu^{J} f(b, a)+f(a, b) \mu=0$ which is satisfied by taking $\mu=(f(a, b))^{-1}$, owing to the relation $f(b, a)=-(f(a, b))^{J}$. The transvection $x \rightarrow u(x)=x+c f(c, x)$ sends then $a$ into $-b \mu$, for $f(c, a)=\mu^{J} f(b, a)$ $=-1$.

Suppose next that $f(a, b)=0$; this means that the plane containing $a$ and $b$ is totally isotropic, hence $n \geqq 3$. Therefore there exists a vector $z$ such that $f(a, z) \neq 0$ and $f(b, z) \neq 0$; the plane containing $a$ and $z$ is hyperbolic, and contains therefore a vector $a_{1}$ not collinear to $a$ and isotropic; moreover $a_{1}$ cannot be orthogonal to $b$, otherwise $z$ would also be orthogonal to $b$; therefore one has $f\left(a, a_{1}\right) \neq 0$ and $f\left(a_{1}, b\right) \neq 0$; applying the preceding result, there is a transvection $u_{1}$ transforming $a$ into a scalar multiple of $a_{1}$, and a transvection $u_{2}$ transforming $a_{1}$ into a scalar multiple of $b$; the transformation $u=u_{2} u_{1}$ satisfies the conditions of the lemma.

6. Our next step will be to prove that:

$2^{\circ}$. Theorem 1 is true for $n=2, \nu \geqq 1$. The assumption implies that there is a basis of $E$ consisting of 2 isotropic vectors $e_{1}, e_{2}$ such that $f\left(e_{1}, e_{2}\right)=1$. If $u$ is a unitary transformation,

$$
U=\left(\begin{array}{ll}
\alpha & \beta \\
\gamma & \delta
\end{array}\right)
$$

its matrix with respect to the basis $\left(e_{1}, e_{2}\right)$, the elements of $U$ satisfy the following conditions

$$
\alpha^{J} \gamma-\gamma^{J} \alpha=0, \quad \beta^{J} \delta-\delta^{J} \beta=0, \quad \alpha^{J} \delta-\gamma^{J} \beta=1,
$$

and conversely, the matrices satisfying these relations are unitary. We observe that from (1) one deduces the following relations

$$
\alpha \beta^{J}-\beta \alpha^{J}=0, \quad \gamma \delta^{J}-\delta \gamma^{J}=0 .
$$

Indeed, let

$$
A=\left(\begin{array}{rr}
0 & 1 \\
-1 & 0
\end{array}\right)
$$

and let $U^{*}$ be the transposed matrix of $U^{J}$; then (1) is equivalent to the matrix relation $U^{*} A U=A$, whence $A^{-1}=U^{-1} A^{-1}\left(U^{*}\right)^{-1}$, and therefore $U A^{-1} U^{*}=A^{-1}$; but as $A^{-1}=-A$, the last relation implies (2) (this short derivation of (2) from (1) was indicated by the referee). The transvections of vector $e_{2}$ have matrices of the type

$$
B(\lambda)=\left(\begin{array}{ll}
1 & 0 \\
1 & 1
\end{array}\right)
$$


where $\lambda \in S$; the transvections of vector $e_{1}$ have matrices of the type

$$
C(\mu)=\left(\begin{array}{ll}
1 & \mu \\
0 & 1
\end{array}\right)
$$

with $\mu \in S$. We want to prove that if a normal subgroup $G$ of $T_{2}$ contains a transformation $u$ not in the center $W_{2}$, then $G=T_{2}$; it will be enough, by virtue of part $1^{\circ}$, to show that all matrices $C(\mu)$ belong to $G$.

Let us first suppose that the matrix $U$ is such that $\beta \neq 0$. Then the matrix

$$
\begin{aligned}
(B(\lambda))^{-1} U B(\lambda) & =B(-\lambda) U B(\lambda) \\
& =\left(\begin{array}{cc}
\alpha+\beta \lambda & \beta \\
\gamma^{\prime} & \delta^{\prime}
\end{array}\right)
\end{aligned}
$$

belongs to $G$, for any $\lambda \in S$. It follows from the first relation (2) that $\beta^{-1} \alpha$ $\in S$; taking $\lambda=-\beta^{-1} \alpha$, we see that we can always limit ourselves to the case in which $\alpha=0$; the third relation (1) then yields $\gamma=-\left(\beta^{-1}\right)^{J}$.

Supposing therefore that $\alpha=0$, we next determine a linear transformation $v$ of $E$ such that $u\left(v\left(e_{1}\right)\right)=e_{1} \xi$, and $v\left(u\left(e_{1}\right)\right)=e_{1} \eta, \xi$ and $\eta$ being at first arbitrary elements $\neq 0$ in $K$. An easy computation shows that the matrix of $v$ with respect to $e_{1}, e_{2}$ is equal to

$$
V=\left(\begin{array}{cc}
-\gamma^{-1} \delta \beta^{-1} \xi & \eta \gamma^{-1} \\
\beta^{-1} \xi & 0
\end{array}\right) .
$$

We now want $v$ to be in the group $T_{2}$; this, by the third condition (1), is possible only if we have

$$
\left(\eta \gamma^{-1}\right)^{J} \beta^{-1} \xi=-1 .
$$

Conversely, if $\xi$ and $\eta$ satisfy (3) and $\beta^{-1} \xi \in S$, then $v \in T_{2}$. To prove this, we first remark that there is $\sigma \in S$ such that

$$
V B(\sigma)=\left(\begin{array}{cc}
0 & -\left(\zeta^{-1}\right)^{J} \\
\zeta & 0
\end{array}\right)
$$

with $\zeta=\beta^{-1} \xi$; indeed, this relation is equivalent to $\sigma=\gamma \eta^{-1} \gamma^{-1} \delta \beta^{-1} \xi$; but it follows from the second relation (2) that $\gamma^{-1} \delta \in S$, and on the other hand, (3) shows that $\gamma \eta^{-1}=-\left(\beta^{-1} \xi\right)^{J}$; therefore, the element $\sigma$ is in $S$.

Further, we have, for $\zeta \in S$,

$$
C\left(-\zeta^{-1}\right) B(\zeta) C\left(-\zeta^{-1}\right)=\left(\begin{array}{cc}
0 & -\zeta^{-1} \\
\zeta & 0
\end{array}\right)
$$

hence $V B(\sigma)$ is in $T_{2}$, which proves that $V$ is in $T_{2}$.

The transformation $u_{1}=u^{-1} v^{-1} u v$ is. then in $G$, and its matrix has the form 


$$
U_{1}=\left(\begin{array}{cc}
\rho & \beta^{\prime} \\
0 & \left(\rho^{-1}\right)^{J}
\end{array}\right),
$$

where $\rho=\beta^{J} \zeta \beta \zeta$. Finally the matrix $W=U_{1} C(\theta) U_{1}^{-1} C(-\theta)$ is in $G$ for every $\theta \in S$, and is equal to

$$
\left(\begin{array}{cc}
1 & \rho \theta \rho^{J}-\theta \\
0 & 1
\end{array}\right)
$$

in other words, it is a matrix $C(\mu)$ with $\mu=\rho \theta \rho^{J}-\theta$.

7. We first want to prove that it is possible to choose $\zeta$ and $\theta$ in the set $S$ of symmetric elements such that $\mu \neq 0$. This will certainly be the case if $\rho \rho^{J} \neq 1$, with $\theta=1$. We have therefore to show that, under the assumptions of Theorem 1 , it is impossible that $\rho \rho^{J}=1$ for every $\zeta \in S$. This is immediate if the subfield $Z_{0}$ of the center $Z$, which consists of the symmetric elements of $Z$ (and is such that $Z$ is a separable quadratic extension of $Z_{0}$, or identical to $Z_{0}$ ), has more than 5 elements; for if $\zeta \in Z_{0}$, the relation $\rho \rho^{J}=1$ reduces tc $\zeta^{4}\left(\beta^{J} \beta\right)^{2}=1$, which can be verified by at most 4 different elements of $Z_{0}$. We are therefore reduced to the case in which $Z_{0}$ has at most 5 elements, which means that $Z$ has at most 25 elements; moreover, we can suppose that $K$ is noncommutative, and therefore infinite. In the identity $\rho \rho^{J}=1$, if we replace $\zeta$ by 1 , we get $\left(\beta^{J} \beta\right)^{2}=1$, hence $\beta^{J}=\beta^{-1}$ or $\beta^{J}=-\beta^{-1}$; in any case, $\beta^{J}$ and $\beta$ commute. If $\beta^{J}+\beta=0$, we have $\beta^{4}=1$; if $\beta+\beta^{J} \neq 0$, we can replace $\zeta$ by $\beta+\beta^{J}$, and we get $\left(\beta+\beta^{J}\right)^{4}=1$. In every case, $\beta$ is a root of an algebraic equation with coefficients in $Z$, and as $Z$ is finite, so is the commutative field $Z(\beta)$. Let $L$ be the subsfield of $K$ consisting of the elements of $K$ which commute with $\beta$; as $Z(\beta)$ has finite degree over $Z, K$ has finite degree over $L$, and therefore $L$ is an infinite sfield [2, p. 104]; moreover, as $Z\left(\beta^{J}\right)=Z(\beta), L$ is invariant under the involution $J$. Now, if we take $\zeta$ in $S \cap L$, the relation $\rho \rho^{J}=1$ reduces to $\zeta^{4}=1$, in other words $\zeta^{2}=1$ or $\zeta^{2}=-1$. If we apply this to $\zeta=\xi+\eta$, where $\xi$ and $\eta$ are arbitrary in $S \cap L$, we conclude that $\xi \eta+\eta \xi$ is in the center $Z$ of $K$, from which it immediately follows that the sfield $M$ generated by $\xi$ and $\eta$ over $Z$ has at most rank 4 over $Z$; as $Z$ is finite, this sfield must be commutative. In other words, any two elements of $S \cap L$ commute; it then follows from Lemma 1 that either $L$ is commutative, or is a reflexive sfield, and then has necessarily an infinite center which is identical to $S \cap L$. In any case, the relation $\zeta^{4}=1$, valid for $\zeta \in S \cap L$ (and $\zeta \neq 0$ ) shows that $S \cap L$ must be finite; this is possible only when $L$ is commutative; but then $S \cap L$ is a subfield of $L$ such that $L$ has degree 2 over $S \cap L$, and as $L$ is infinite, $S \cap L$ would also have to be infinite; we thus have reached a contradiction, which ends this part of the argument.

8. We now have proved that there exists in $S$ an element $\mu_{0} \neq 0$ such that $C\left(\mu_{0}\right)$ belongs to $G$. We want to show next that $C(1)$ also belongs to $G$. In order to do this, we repeat the whole argument of $\$ \$ 6$ and 7 , starting with 
the matrix $C\left(\mu_{0}\right)$ instead of $U$, and, therefore, this time the element $\beta=\mu_{0}$ is symmetric. If we can take $\zeta$ in the center $Z$, we thus get an element $\rho$ which is symmetric and such that $\rho^{2} \neq 1$. If not, which is the case only when $Z_{0}$ has at most 5 elements, the commutative field $Z(\beta)$ is either finite or infinite. If it is infinite, we can again take a symmetric $\zeta$ in $Z(\beta)$ such that $\rho$ is symmetric and $\rho^{2} \neq 1$. If on the contrary $Z(\beta)$ is finite, an argument similar to that of $\S 7$, where $Z(\beta)$ replaces $Z$, proves that in the subsfield $L$ of $K$ commuting with $\beta$ it is possible to find a symmetrical element $\zeta$ such that $\zeta^{4} \beta^{4} \neq 1$, and then $\rho=\beta^{2} \zeta^{2}$ is again symmetric and such that $\rho^{2} \neq 1$. Now, in the method of $\S 6$, we can take $\theta=\left(\rho^{2}-1\right)^{-1}$; then $\rho$ and $\theta$ commute, and the matrix we obtain in that way is $C(1)$.

Finally, let $\mu$ be any symmetric element $\neq 0$, and consider the subsfield $N$ of $K$ commuting with $\mu$; we are going to prove that there exists in $N$ a symmetric element $\zeta$ such that $\zeta^{4} \neq 1$. This is certainly the case if the center of $N$ (which contains the commutative field $Z(\mu)$ ) is infinite (or has more than 25 elements). On the other hand, if the center of $N$ is finite and is distinct from $N$, in particular $Z(\mu)$ is finite, and then $N$ is necessarily infinite; but then the argument of $\$ 7$ shows that it is impossible that $\zeta^{4}=1$ for every symmetric element in $N$. The symmetric element $\zeta$ being thus chosen, we apply again the procedure of $\$ 6$, starting this time from the matrix $C(1)$ instead of $U$; we take then $\rho=\zeta^{2}$, and $\rho$ is symmetric and such that $\rho^{2} \neq 1$. Moreover, $\rho$ commutes with $\mu$ and with $\zeta$ (which commute together); therefore, if we take this time $\theta=\mu\left(\zeta^{4}-1\right)^{-1}, \theta$ is symmetric, and we have $\rho \theta \rho^{J}$ $-\theta=\mu$.

9. To end the proof of step $2^{\circ}$, we still have to consider the cases in which $\beta=0$ in the matrix $U$. Suppose first that $\gamma \neq 0$; then, if

$$
Q=\left(\begin{array}{rr}
0 & -1 \\
1 & 0
\end{array}\right)
$$

we notice that $Q=C(-1) B(1) C(-1)$ belongs to $T_{2}$ and that

$$
Q U Q^{-1}=\left(\begin{array}{rr}
\delta & -\gamma \\
0 & \alpha
\end{array}\right),
$$

and we are reduced to the preceding case. Finally, if $\beta=\gamma=0$, we have $\delta=\left(\alpha^{-1}\right)^{J}$ by the third relation (1); then the matrix $C(\mu) U C(-\mu)$ belongs to $G$, and it is equal to

$$
\left(\begin{array}{cc}
\alpha & \mu\left(\alpha^{-1}\right)^{J}-\alpha \mu \\
0 & \left(\alpha^{-1}\right)^{J}
\end{array}\right) .
$$

We are therefore reduced to the former case if there is a symmetric $\mu$ such that $\mu\left(\alpha^{-1}\right)^{J}-\alpha \mu \neq 0$. If not, $U$ commutes with every matrix $C(\mu)$, and it is easily verified that it also commutes with every matrix $B(\lambda)$. But this is 
possible only if $U$ is in the center $W_{2}$ of $T_{2}$, owing to the following lemma:

LеммA 3. The group $T_{2}$ is generated by the transvections $B(\lambda)$ and $C(\mu)$.

To prove that lemma, consider an arbitrary isotropic vector $x=e_{1} \alpha+e_{2} \beta$ in $E$; one has then $\alpha^{J} \beta-\beta^{J} \alpha=0$. Suppose $\beta \neq 0$; then $\alpha \beta^{-1}$ is a symmetric element. But then the transvection $C(\mu)$, with $\mu=-\alpha \beta^{-1}$, transforms $x$ into a vector collinear with $e_{2}$, and this shows that every transvection of vector $x$ is transformed by $C(\mu)$ into a transvection of vector $e_{2}$, that is, a transvection $B(\lambda)$. This of course proves the lemma, and ends the proof of step $2^{\circ}$ of Theorem 1.

10. It is now easy to prove that Theorem 1 is true for any $n \geqq 3$. Let $G$ be a normal subgroup of $T_{n}$, and $u$ a transformation in $G$ which does not belong to the center $W_{n}$. Then $u$ does not belong to $Z_{n}$, in other words it is not a homothetic mapping. From that, we shall deduce that there exists an isotropic vector $x$ such that $u(x)$ and $x$ are not collinear. This will be proved if we show that when $u$ leaves invariant every isotropic line, it leaves invariant every line (and is therefore a homothetic mapping), according to the following lemma:

Lемма 4. For $n \geqq 3$ and $\nu \geqq 1$, every nonisotropic line in $E$ is the intersection of two hyperbolic planes.

To prove the lemma, let $x$ be a nonisotropic vector, and $y$ an isotropic vector. Let $z$ be a vector which is orthogonal neither to $x$ nor to $y$ and is not in the plane determined by $x$ and $y$. Then the plane $P$ determined by $y$ and $z$ is a hyperbolic plane, and it contains therefore a second isotropic vector $y_{1}$ such that $f\left(y, y_{1}\right)=1$. Moreover, any vector $y_{2}=y \alpha+y_{1} \beta$ is isotropic if $\alpha^{J} \beta-\beta^{J} \alpha=0$, and therefore there exists such a vector $y_{2}$ which is collinear with neither of $y$ and $y_{1}$ (take for instance $\alpha=\beta=1$ ). Among the three isotropic vectors $y, y_{1}, y_{2}$, two at least are not orthogonal to $x$, since $x$ is not orthogonal to $P$. Therefore two of the three planes $Q, Q_{1}, Q_{2}$ determined by $x$ and the vectors $y, y_{1}, y_{2}$, respectively, are hyperbolic planes, which proves the lemma.

We can now resume the end of the proof of Theorem 1. Let $x$ be an isotropic vector such that $x$ and $u(x)$ are not collinear. Suppose first that $f(x, u(x))=0$. Then there exists a vector $z$ which is orthogonal to $u(x)$ but not to $x$. The plane $P$ determined by $x$ and $z$ is a hyperbolic plane, hence contains an isotropic vector $y$ which is not collinear to $x$. From Lemma 2, there exists a transvection $v \in T_{n}$ transforming $x$ into a scalar multiple $y \lambda$ of $y$; moreover the vector of that transvection is in $P$, hence orthogonal to $u(x)$, and therefore $v(u(x))=u(x)$. The transformation $u_{1}=v u^{-1} v^{-1} u$ belongs to $G$, and one has $u_{1}(x)=y$. This proves that we can always suppose that $u \in G$ is such that $f(x, u(x)) \neq 0$.

Let then $w$ be a transvection of vector $x$; $u w u^{-1}$ is a transvection of vector 
$u(x)$, and as $x$ and $u(x)$ are not collinear, these two transvections do not commute. Let $Q$ be the hyperbolic plane determined by $x$ and $u(x)$; the transformation $u_{2}=w^{-1} u w u^{-1}$ belongs to $G$, and leaves invariant every vector in the subspace $Q^{*}$ orthogonal to $Q$. It therefore belongs to the subgroup $\Gamma$ of $U_{n}(K, f)$ which leaves invariant every vector of $Q^{*}$, and is obviously isomorphic to the unitary group $U_{2}\left(K, f_{1}\right)$, where $f_{1}$ is the restriction of $f$ to the plane $Q$; we shall identify $\Gamma$ with that group. Moreover, $u_{2}$ is the product of two transvections, hence belongs to the group $T_{2}\left(K, f_{1}\right)$; finally, it is not in the center of that group, since it does not commute with $w$. Now step $2^{\circ}$ of the proof shows that $G$ contains every transformation of $T_{2}\left(K, f_{1}\right)$, in particular every transvection of vector $x$. Applying step $1^{\circ}$ of the proof, we see that $G=T_{n}$, and Theorem 1 is completely proved.

11. We can supplement Theorem 1 by proving the following theorem.

THEOREM 2. Under the same assumptions as in Theorem 1, the center $W_{n}$ of the group $T_{n}$ is the intersection $T_{n} \cap Z_{n}$.

Indeed, if $n \geqq 3$, every transformation $u \in W_{n}$ must commute with every transvection, hence leave invariant every isotropic line. It then follows from Lemma 4 that $u$ leaves invariant every line, hence is a homothetic mapping.

For $n=2$, if $e_{1}$ and $e_{2}$ are two isotropic vectors constituting a basis of $E$ such that $f\left(e_{1}, e_{2}\right)=1$, the matrix

$$
U=\left(\begin{array}{ll}
\alpha & \beta \\
\gamma & \delta
\end{array}\right)
$$

of $u$ with respect to that basis must commute with every one of the matrices $B(\lambda)$ and $C(\mu)$ (notations of $\S 6$ ); this, as is readily seen, means that

$$
U=\left(\begin{array}{cc}
\alpha & 0 \\
0 & \left(\alpha^{-1}\right)^{J}
\end{array}\right),
$$

where $\alpha$ is such that $\alpha \lambda=\lambda\left(\alpha^{-1}\right)^{J}$ for every symmetric element $\lambda \in K$. Taking $\lambda=1$ gives $\alpha^{J}=\alpha^{-1}$, and therefore $\alpha$ must commute with every symmetric element. From Lemma 1, we deduce therefore that $\alpha$ is in the center $Z$ of $K$ (and therefore that $u \in T_{2} \cap Z_{2}$ ) with the possible exception of the case in which $K$ is a reflexive sfield of characteristic $\neq 2$, and $Z$ is identical to the set $S$ of symmetric elements. But in that case we remark that the matrices $B(\lambda)$ and $C(\mu)$ have their elements in $Z$, and from Lemma 3 it follows that the same is true for every matrix of the group $T_{2}$; hence if the matrix $U$ belongs to $T_{2}, \alpha$ is again in $Z$, and this ends the proof of Theorem 2 .

12. The remainder of this paper is devoted to the study of the quotient group $U_{n} / T_{n}$; the results we obtained in that direction are far from complete, and part of them are valid only under the additional assumption that the sfield $K$ has finite rank over its center $Z$. 
We begin by proving a lemma which is valid for any sfield $K$. A plane rotation is a transformation $u \in U_{n}$ which leaves invariant every element of a nonisotropic $(n-2)$-dimensional subspace $Q$; the plane $Q^{*}$ orthogonal to $Q$ is then called the plane of the rotation $u$. A hyperbolic rotation is a plane rotation whose plane is hyperbolic. We then prove the following lemma.

LEMмA 5. For $\nu \geqq 1$, every unitary transformation is a product of hyperbolic rotations.

The lemma being obvious for $n=2$, we prove it by induction on $n$, as in $[5$, p. 66]. Let $u$ be any unitary transformation, and let $x$ be a nonisotropic vector such that the hyperplane $H$ orthogonal to $x$ contains isotropic vectors. If $u(x)=x, u$ leaves $H$ invariant, and we can apply induction to its restriction to $H$, since the index of the restriction of the form $f$ to $H$ is $\geqq 1$ by assumption; the lemma is then proved. If $u(x) \neq x$, there is always a hyperbolic plane $P$ containing the vector $u(x)-x$ : indeed, if $a=u(x)-x$ is not isotropic, there is an isotropic vector $b$ not orthogonal to $a$ (Lemma 4 ), and then the plane $P$ determined by $a$ and $b$ is hyperbolic; if on the contrary $a$ is isotropic, there is a nonisotropic vector $c$ not orthogonal to $a$, and the plane $P$ determined by $a$ and $c$ is hyperbolic. Now, as $u(x)-x$ is in $P$, we can write $x=z+y$, $u(x)=z+y^{\prime}$, where $y$ and $y^{\prime}$ are in $P$, and $z$ in the $(n-2)$-dimensional subspace $P^{*}$ orthogonal to $P$. Moreover, as $f(u(x), u(x))=f(x, x)$, we have also $f(y, y)=f\left(y^{\prime}, y^{\prime}\right)$. From Witt's theorem applied to the restriction of $f$ to the plane $P$, it follows that there exists a plane rotation $v$ of plane $P$ such that $v(y)=y^{\prime}$, hence also $v(x)=u(x)$, since $v(z)=z$. But then $v^{-1} u$ leaves $x$ invariant, and we are reduced to the first case: $v^{-1} u$ is thus a product of hyperbolic rotations, and so is therefore $u$.

13. We shall use Lemma 5 to prove that in certain cases the subgroup $T_{n}$ is identical to $U_{n}$ : Lemma 5 shows that this will be done if we can prove that every hyperbolic rotation is a product of transvections. In particular, we shall have proved that $U_{n}=T_{n}$ for every dimension $n$ if we can prove that $U_{2}=T_{2}$ (for $\nu \geqq 1$, of course). We therefore begin by investigating the relations between the group $U_{2}$ and its subgroup $T_{2}$.

As in $\$ 6$, we consider a basis of $E$ consisting of two isotropic vectors $e_{1}, e_{2}$ such that $f\left(e_{1}, e_{2}\right)=1$; let

$$
U=\left(\begin{array}{ll}
\alpha & \beta \\
\gamma & \delta
\end{array}\right)
$$

be the matrix of a unitary transformation $u$ with respect to that basis; the relations (1) and (2) are then satisfied. As $\alpha$ and $\beta$ are not both 0 , there is a $\sigma \in S$ such that in

$$
U B(\sigma)=\left(\begin{array}{ll}
\alpha^{\prime} & \beta^{\prime} \\
\gamma^{\prime} & \delta^{\prime}
\end{array}\right),
$$


$\alpha^{\prime}=\alpha+\beta \sigma \neq 0$; we can therefore already suppose that $\alpha \neq 0$; then it follows from the first relation (2) that $\mu=\alpha^{-1} \beta$ and from the first relation (1) that $\lambda=\gamma \alpha^{-1}$ are both symmetric. But then the matrix

$$
B(-\lambda) U C(-\mu)=\left(\begin{array}{cc}
\alpha & 0 \\
0 & \left(\alpha^{-1}\right)^{J}
\end{array}\right)
$$

(owing to the third relation (1)). If we observe that $T_{2}$ is a normal subgroup of $U_{2}$, and that $T_{2}$ is generated by the matrices $B(\xi)$ and $C(\eta)$ (Lemma 3 ), we finally see that every matrix $U$ in the group $U_{2}$ can be written as a product $V W$, where $W$ belongs to the group $T_{2}$, and $V$ has the form

$$
\left(\begin{array}{cc}
\alpha & 0 \\
0 & \left(\alpha^{-1}\right)^{J}
\end{array}\right)
$$

In order that $T_{2}=U_{2}$, it is therefore necessary and sufficient that every matrix

$$
\left(\begin{array}{cc}
\alpha & 0 \\
0 & \left(\alpha^{-1}\right)^{J}
\end{array}\right)
$$

belong to $T_{2}$. Now, for every pair of elements $\lambda, \mu$ in $S$, we have

$$
C(\mu) B(\lambda)=\left(\begin{array}{cr}
1+\mu \lambda & \mu \\
\lambda & 1
\end{array}\right)
$$

if we apply the preceding method to that matrix, we see that every matrix

$$
\left(\begin{array}{cc}
\alpha & 0 \\
0 & \left(\alpha^{-1}\right)^{J}
\end{array}\right)
$$

with $\alpha=1+\mu \lambda=\left(\lambda^{-1}+\mu\right) \lambda$ belongs to $T_{2}$.

This proves that $T_{2}=U_{2}$ if every element $\neq 0$ in $K$ is a product of elements of $S$.

14. Let us suppose in this section that $K$ has finite rank $m^{2}$ over its center $Z$. We recall that $K$ is said to be of the first kind if $J$ leaves invariant every element of $Z$, of the second kind if the restriction of $J$ to $Z$ is not the identity (it is then an involution in $Z$ ). Moreover, when $K$ is of the first kind and of characteristic $\neq 2$, the dimension of $S$ over $Z$ is equal to $m(m+1) / 2$ or $m(m-1) / 2$ [7]; the easiest way to see this is to extend $Z$ to a splitting field $L$ of $K$; the involution $J$ is extended to $K_{(L)}$ in an obvious way (the elements of $L$ being invariant by $J$ ), and by taking a basis of $K$ over $Z$ consisting of symmetric or skew-symmetric elements, one sees readily that the dimension over $L$ of the space of symmetric elements of $K_{(L)}$ is equal to the dimension over $Z$ of the space of symmetric elements of $K$. But $K_{(L)}$ is the algebra of matrices of order $m$ over $L$, and an involution of that alzebra leaving in- 
variant the elements of $L$ is known, namely the mapping $X \rightarrow^{t} X$, where ${ }^{t} X$ is the transposed matrix of $X$; therefore $[1, \mathrm{p} .896]$, one has $X^{J}=P^{-1 . t} X \cdot P$, where $P$ is either a symmetric or a skew-symmetric matrix. Hence, the relation $X^{J}=X$ means that $P X$ is symmetric (resp. skew-symmetric) if $P$ is symmetric (resp. skew-symmetric); this proves at once our assertion. Similarly, it is shown that when the characteristic of $K$ is 2, the dimension of $S$ over $Z$ is always $m(m+1) / 2$ when $K$ is of the first kind.

We can now prove the following theorem.

THEOREM 3. When $K$ is a sfield of the first kind, of finite rank $m^{2}$ over its center $Z$ and of characteristic $\neq 2$, and such that the space $S$ of symmetric elements in $K$ has dimension $m(m+1) / 2$ over $Z$, then $U_{n}=T_{n}$ for every $n \geqq 2$.

All we have to prove (according to the final remark of $\S 13$ ) is that, for every $\zeta \in K$, there exist two elements $\xi$, $\eta$ in $S$ such that $\zeta=\xi \eta$. If $\theta=\eta^{-1}$, this amounts to saying that there exists an element $\theta \in S$ such that $\zeta \theta$ is symmetric, which means that $\zeta \theta-\theta \zeta^{J}=0$. But the mapping $\theta \rightarrow \zeta \theta-\theta \zeta^{J}$ of $S$ into $K$ is linear with respect to $Z$, and maps $S$ into the space $A$ of skew-symmetric elements, which is supplementary to $S$ in $K$, hence has a dimension equal to $m(m-1) / 2$; as $m(m+1) / 2>m(m-1) / 2$, the kernel of the linear mapping $\theta \rightarrow \zeta \theta-\theta \zeta^{J}$ is not reduced to 0 , and this ends our proof.

As a corollary, we obtain Theorem 6 of [5] when $K$ is a reflexive sfield of characteristic $\neq 2$ : the passage from an hermitian to a skew-hermitian form over $K$, explained in $\S 3$, replaces the involution $\xi \rightarrow \bar{\xi}$ in $K$ by an involution for which the symmetric elements are the skew-symmetric elements of $\xi \rightarrow \xi$, hence form a subspace of dimension 3 over the center $Z$.

15. Turning now to the case in which the sfield $K$, of finite rank $m^{2}$ over $Z$, is a sfield of the first kind but such that $S$ has dimension $m(m-1) / 2$ over $Z$ (this property implying that $K$ has a characteristic $\neq 2$ ), we have to set aside the case $m=2$, in which $S=Z$, and therefore $S$ cannot generate the group $K^{*}$ of elements $\neq 0$ in $K$. When $m>2$, it seems likely (due to Lemma 1 ) that $S$ generates $K^{*}$, but $\mathrm{I}$ have not been able to prove that conjecture, and in the absence of any further assumptions, the structure of the group $U_{n} / T_{n}$ remains unknown in that case. I shall therefore consider only the case $m=2$; in other words, $K$ is then a sfield of generalized quaternions over $Z$, and the involution $J$ is the (unique) involution of $K$ for which the elements of $Z$ are the only symmetric elements.

Let us first consider the case $n=2$; then $T_{2}$ is simply the unimodular group $S L_{2}(Z)[4$, p. 30]. Moreover, as every element $\alpha \in K$ is such that $\left(\alpha^{-1}\right)^{J}=\alpha \cdot(N(\alpha))^{-1}$, where $N(\alpha)=\alpha \alpha^{J} \in Z$, it follows from $\$ 13$ that every matrix $U$ in the group $U_{2}$ can be written $\alpha X$, where $X$ is an arbitrary matrix in $G L_{2}(Z)$ such that $\operatorname{det}(X)=(N(\alpha))^{-1}$, and $\alpha$ is an arbitrary element in $K^{*}$. We observe in addition that $\alpha$ and $X$ are permutable, and that $\alpha$ is determined by $U$ up to a factor $\lambda \in Z^{*}$ (the matrix $X$ being then multiplied 
by $\lambda^{-1}$ ). We can therefore describe the structure of the group $U_{2}$ in the following way: consider in the direct product $K^{*} \times G L_{2}(Z)$ the subgroup $\mathrm{I}$ consisting of the pairs $(\alpha, X)$ such that $N(\alpha) \cdot \operatorname{det}(X)=1$, and let $\Delta$ be the subgroup of $\Gamma$ consisting of the pairs $\left(\lambda, \lambda^{-1}\right)$, where $\lambda \in Z^{*}$; then $U_{2}$ is isomorphic to the factor group $\Gamma / \Delta$. We observe that $U_{2}$ contains as a normal subgroup the multiplicative group $U_{1}$ of elements of norm 1 in $K$, and that $U_{1}$ and $T_{2}$ commute and have as their intersection the two elements 1 and -1 , which constitute the center $W_{2}$ of $T_{2}$; the quotient group $U_{2} / T_{2}$ contains $U_{1} / W_{2}$ as a subgroup, hence $T_{2}$ is certainly not the commutator subgroup of $U_{2}$.

16. There are reasons to believe that the preceding structure of the group $U_{2}(K, f)$ when $K$ is a sfield of generalized quaternions and $f$ a skew-hermitian form is exceptional among the corresponding groups $U_{n}(K, f)$ for $n>2$, much as the 4-dimensional orthogonal groups among the orthogonal groups of other dimensions. The evidence I can supply in favor of that view is summed up in the following theorem:

THeOREM 4. If $K$ is a sfield of characteristic $\neq 2$, and the index $\nu$ of the form $f$ is at least 2 (which implies $n \geqq 4$ ), then $T_{n}$ is the commutator subgroup of $U_{n}(K, f)$.

To prove that theorem, we shall establish two lemmas.

Lemma 6. Let $P$ be a hyperbolic plane, $\Gamma$ the group of hyperbolic rotations of plane $P$. Then (for $\nu \geqq 2)$ the factor group $\Gamma /\left(\Gamma \cap T_{n}\right)$ is abelian.

Let $e_{1}, e_{2}$ be two isotropic vectors forming a basis of $P$, with $f\left(e_{1}, e_{2}\right)=1$; it is then possible to find two other isotropic vectors $e_{3}, e_{4}$ orthogonal to $P$ and such that $f\left(e_{3}, e_{4}\right)=1$ (because $\nu \geqq 2$ ). Let $Q$ and $R$ be the totally isotropic planes determined by $e_{1}, e_{3}$ and $e_{2}, e_{4}$ respectively; if $u \in U_{n}$ leaves invariant both planes $Q$ and $R$, and $V$ and $W$ are the matrices of the restrictions of $u$ to $Q$ and $R$, with respect to the bases $e_{1}, e_{3}$ and $e_{2}, e_{4}$ respectively, one has $W=\left(V^{\prime}\right)^{J}, V^{\prime}$ being the contragredient of $V$. We are going to prove that there are transformations $u \in T_{n}$ of the preceding type, and such that $V=B(\lambda)$, where $\lambda$ is any element of $K$. Let $a=e_{2} \alpha+e_{3} \beta$ be any vector in the totally isotropic plane determined by $e_{2}$ and $e_{3}$, and consider the transvection $w$ such that $w(x)=x+a f(a, x)$; it leaves invariant $e_{2}$ and $e_{3}$, and is such that

$$
w\left(e_{1}\right)=e_{1}-e_{2} \alpha \alpha^{J}-e_{3} \beta \alpha^{J}, \quad w\left(e_{4}\right)=e_{4}+e_{2} \alpha \beta^{J}+e_{3} \beta \beta^{J} .
$$

Let $a_{1}=e_{2} \alpha_{1}+e_{3} \beta_{1}$ be a second isotropic vector, $w_{1}$ the transvection such that $w_{1}(x)=x-a_{1} f\left(a_{1}, x\right)$; then $u=w_{1} w$ leaves invariant $e_{2}$ and $e_{3}$ and is such that

$$
\begin{aligned}
& u\left(e_{1}\right)=e_{1}+e_{2}\left(\alpha_{1} \alpha_{1}^{J}-\alpha \alpha^{J}\right)+e_{3}\left(\beta_{1} \alpha_{1}^{J}-\beta \alpha^{J}\right), \\
& u\left(e_{4}\right)=e_{4}+e_{2}\left(\alpha \beta^{J}-\alpha_{1} \beta_{1}\right)+e_{3}\left(\beta \beta^{J}-\beta_{1} \beta_{1}^{J}\right) .
\end{aligned}
$$


If we take $\alpha_{1}=\alpha$ and $\beta_{1}=-\beta, u$ leaves invariant $Q$ and $R$, and is such that $u\left(e_{1}\right)=e_{1}-2 e_{3} \beta \alpha^{J}$; as the characteristic of $K$ is not 2 , it is possible to take $\alpha$ and $\beta$ such that $-2 \beta \alpha^{J}=\lambda$, for any element $\lambda \in K$, and the matrix of the restriction of $u$ to $Q$ is then $B(\lambda)$. Similarly, it can be proved that $u \in T_{n}$ exists such that $V=C(\mu)$ for any $\mu \in K$. Therefore $T_{n}$ contains all the transformations $u \in U_{n}$ leaving invariant $Q$ and $R$ and such that the matrix of the restriction of $u$ to $Q$ is any matrix $V$ in the unimodular group $S L_{2}(K)$ [4, p. 30]; in particular, for any element $\gamma$ in the commutator subgroup of $K^{*}, u \in T_{n}$ exists such that

$$
V=\left(\begin{array}{ll}
\gamma & 0 \\
0 & 1
\end{array}\right),
$$

$[4$, p. 29], which means that $u$ is a hyperbolic rotation of plane $P$, such that its matrix in $P$ is

$$
\left(\begin{array}{cc}
\gamma & 0 \\
0 & \left(\gamma^{-1}\right)^{J}
\end{array}\right) .
$$

Now we have seen in $\S 13$ that every hyperbolic rotation of plane $P$ has a matrix (with respect to $e_{1}, e_{2}$ ) which can be written as the product of a matrix

$$
\left(\begin{array}{cc}
\alpha & 0 \\
0 & \left(\alpha^{-1}\right)^{J}
\end{array}\right)
$$

(with $\alpha \in K^{*}$ ) and a matrix of $\Gamma \cap T_{n}$. If, to every $\alpha \in K^{*}$, we associate the class of the matrix

$$
\left(\begin{array}{cc}
\alpha & 0 \\
0 & \left(\alpha^{-1}\right)^{J}
\end{array}\right),
$$

modulo the subgroup $\Gamma \cap T_{n}$, we define a homomorphism of $K^{*}$ onto $\Gamma /\left(\Gamma \cap T_{n}\right)$, and the preceding result shows that the kernel of that homomorphism contains the commutator subgroup $C$ of $K^{*}$; hence $\Gamma /\left(\Gamma \cap T_{n}\right)$ is isomorphic to a quotient group of the abelian group $K^{*} / C$.

17. Lemma 7. Let $P_{1}$ and $P_{2}$ be any two hyperbolic planes. Then (for $\left.\nu \geqq 2\right)$ there exists a transformation $w \in T_{n}$ such that $w\left(P_{1}\right)=P_{2}$.

It follows from Lemma 2 that there exists a transformation in $T_{n}$ sending an isotropic vector in $P_{1}$ into an isotropic vector in $P_{2}$; we can therefore assume in the following proof that there exists a common isotropic vector $e_{2}$ in $P_{1}$ and $P_{2}$. We now consider separately several cases.

(a) The dimension $n=4$. Let $e_{1}$ be a second isotropic vector in $P_{1}$ such that $f\left(e_{1}, e_{2}\right)=1$, and let $e_{3}, e_{4}$ be determined as in the proof of Lemma 6 . There exists in $P_{2}$ an isotropic vector $e_{1}^{\prime}$ such that $f\left(e_{1}^{\prime}, e_{2}\right)=1$; we can write $e_{1}^{\prime}=e_{1}$ 
$+e_{2} \beta+e_{3} \gamma+e_{4} \delta$, and the condition $f\left(e_{1}^{\prime}, e_{1}^{\prime}\right)=0$ is equivalent to

$$
\beta-\beta^{J}+\gamma^{J} \delta-\delta^{J} \gamma=0
$$

which can be written $\beta+\gamma^{J} \delta=\left(\beta+\gamma^{J} \delta\right)^{J}$, and means therefore that the expression $\beta+\gamma^{J} \delta$ is a symmetric element $\lambda$. Now, it has been proved in the proof of Lemma 6 that the transformation $w_{1}$ leaving invariant $e_{2}$ and $e_{3}$, and such that

$$
w_{1}\left(e_{1}\right)=e_{1}+e_{3} \gamma, \quad w_{1}\left(e_{4}\right)=e_{4}-e_{2} \gamma^{J},
$$

belongs to $T_{n}$. Similarly (exchanging the parts played by $e_{3}$ and $e_{4}$ ), the transformation $w_{2}$ leaving invariant $e_{2}$ and $e_{4}$, and such that

$$
w_{2}\left(e_{1}\right)=e_{1}+e_{4} \delta, \quad w_{2}\left(e_{3}\right)=e_{3}-e_{2} \delta^{J},
$$

belongs to $T_{n}$. The transformation $w_{1} w_{2}$, which belongs to $T_{n}$, is such that $w_{1} w_{2}\left(e_{2}\right)=e_{2}$, and $w_{1} w_{2}\left(e_{1}\right)=e_{1}+e_{3} \gamma+e_{4} \delta-e_{2} \gamma^{J} \delta$. Let finally $v$ be the transvection $x \rightarrow x-e_{2} \lambda f\left(e_{2}, x\right)$, which leaves invariant $e_{2}, e_{3}, e_{4}$ and is such that $v\left(e_{1}\right)=e_{1}+e_{2} \lambda$; the transformation $w=v w_{1} w_{2}$ belongs to $T_{n}$, leaves $e_{2}$ invariant, and is such that

$$
w\left(e_{1}\right)=e_{1}+e_{2}\left(\lambda-\gamma^{J} \delta\right)+e_{3} \gamma+e_{4} \delta=e_{1}^{\prime} .
$$

Therefore $w\left(P_{1}\right)=P_{2}$, and the lemma is proved in that case.

(b) $n>4$ and the 3-dimensional subspace $M=P_{1}+P_{2}$ is isotropic. This means that there exists in $M$ at least an isotropic vector $c$ orthogonal to $M$; such a vector cannot be in $P_{1}$, since $P_{1}$ is not isotropic. Therefore the three vectors $c, e_{1}, e_{2}$ ( $e_{1}$ being defined as in (a)) constitute a basis for $M$, such that $f\left(e_{1}, e_{2}\right)=1, f\left(e_{1}, c\right)=f\left(e_{2}, c\right)=0$. There exists then in $E$ a fourth isotropic vector $d$ such that $f(c, d)=1, f\left(e_{1}, d\right)=f\left(e_{2}, d\right)=0[5$, p. 18], and the four vectors $e_{1}, e_{2}, c, d$ form the basis of a nonisotropic 4-dimensional subspace $N$ of $E$ containing $P_{1}$ and $P_{2}$ and such that the restriction of the form $f$ to $N$ has an index equal to 2 . The result of case (a) proves then the lemma.

(c) $n>4$ and the space $M$ is not isotropic. There exists then in $M$ a nonisotropic vector $c$ orthogonal to $P_{1}$. As the index $\nu \geqq 2$, the restriction of $f$ to the $(n-2)$-dimensional subspace $P_{1}^{*}$ orthogonal to $P_{1}$ has an index $\geqq 1$, by Witt's theorem. Therefore (Lemma 4), there exists a hyperbolic plane $Q$ contained in $P_{1}^{*}$ and containing $c$. The subspace $N=P_{1}+Q$ is then a nonisotropic 4-dimensional subspace of $E$, such that the restriction of $f$ to $N$ has index 2 , and $N$ contains $P_{1}$ and $P_{2}$. The proof of the lemma then follows as in case (b).

18. To end the proof of Theorem 4, let us consider a fixed hyperbolic plane $P$. We are going to show that every unitary transformation $v$ can be written $s u$, where $s$ is a hyperbolic rotation of plane $P$, and $u$ belongs to $T_{n}$. The result is true if $v$ is a hyperbolic rotation of plane $P^{\prime}$, for by Lemma 7 there exists $t \in T_{n}$ such that $t(P)=P^{\prime}$, and therefore $v=t s t^{-1}$, where $s$ is a 
rotation of plane $P$; but we can also write $v=s\left(s^{-1} t s\right) t^{-1}$, and as $T_{n}$ is a normal subgroup, $s^{-1} t s \in T_{n}$. Suppose now that $v$ is a product of $p$ hyperbolic rotations (Lemma 5), and use induction on $p$. Let $v=w_{1} w_{2}$, where $w_{1}$ is a hyperbolic rotation and $w_{2}$ is a product of $p-1$ hyperbolic rotations; we can write by assumption $w_{1}=s_{1} u_{1}, \quad w_{2}=s_{2} u_{2}$, hence $v=s_{1} u_{1} s_{2} u_{2}=s_{1} s_{2}\left(s_{2}^{-1} u_{1} s_{2}\right) u_{2}$, and this proves our contention. We have thus shown that the group $U_{n} / T_{n}$ is isomorphic to $\Gamma /\left(\Gamma \cap T_{n}\right)$, hence abelian (and isomorphic to a quotient group of $\left.K^{*} / C\right)$. Theorem 4 then follows from the fact that $T_{n} / W_{n}$ is a simple group (Theorem 1).

19. In special cases it is possible to obtain more precise information. Let us suppose for instance that $K$ is the sfield of ordinary quaternions over a Euclidean ordered field $Z$ (i.e., an ordered field in which every positive element has a square root in $Z$ ). The usual theory of quaternions can then be carried out exactly as when $Z$ is the field $R$ of real numbers; we know therefore that every quaternion $\xi \neq 0$ can be written in one and only one way $\xi=\rho \zeta$, where $\rho \in Z, \rho>0$, and $\rho^{2}=N(\xi)$, hence $N(\zeta)=1$; moreover, every quaternion of norm 1 is a commutator; finally, if $\xi$ and $\eta$ are two quaternions of norm 1 and scalar 0 , there is a third quaternion $\alpha$ of norm 1 such that $\xi=\alpha \eta \alpha^{-1}$. We suppose as usual that $J$ is the only involution in $K$ leaving invariant the elements of $Z$, and that $f$ is skew-hermitian. We can then show that there exists an orthogonal basis in $E$ with respect to which $f(x, y)=\sum_{k=1}^{n} \xi_{k}^{J} i_{k}$. Indeed, there exists an orthogonal basis $\left(e_{k}\right)$ for $f$, and with respect to that basis, $f(x, y)=\sum_{k=1}^{n} \xi_{k}^{J} \alpha_{k} \xi_{k}$, with $\alpha_{k}^{J}=-\alpha_{k}$, which means that the scalar of the quaternion $\alpha_{k}$ is 0 . We can write $\alpha_{k}=\rho_{k} \beta_{k}$, with $\rho_{k}>0, N\left(\beta_{k}\right)=1$, and $\beta_{\mathbf{k}}^{J}=-\beta_{k}$, and therefore $\alpha_{k}=\rho_{k} \gamma_{k} i \gamma_{\mathbf{k}}^{-1}$, where $N\left(\gamma_{k}\right)=1$, hence $\gamma_{\boldsymbol{k}}^{J}=\gamma_{\boldsymbol{k}}^{-1}$. If we replace $e_{k}$ by $e_{k}\left(\rho^{1 / 2}\right)^{-1} \gamma_{k}$, we obtain for $f(x, y)$ the canonical expression $\sum_{k=1}^{n} \xi_{k}^{J} i \xi_{k}$. This proves that all nondegenerate skew-hermitian forms over $E$ are equivalent, hence their index is $[n / 2]$. In particular, for $n \geqq 4, \nu \geqq 2$, and therefore Theorem 4 applies. But here every matrix

$$
\left(\begin{array}{cc}
\alpha & 0 \\
0 & \left(\alpha^{-1}\right)^{J}
\end{array}\right)
$$

can be written

$$
\left(\begin{array}{cc}
\gamma & 0 \\
0 & \left(\gamma^{-1}\right)^{J}
\end{array}\right)\left(\begin{array}{cc}
\rho & 0 \\
0 & \rho^{-1}
\end{array}\right),
$$

where $N(\gamma)=1$, hence $\gamma$ is a commutator, and $\rho \in Z$; as the matrix

$$
\left(\begin{array}{cc}
\rho & 0 \\
0 & \rho^{-1}
\end{array}\right)
$$

belongs to $S L_{2}(Z)$, the proof of Lemma 6 shows that we have here $\Gamma=\Gamma \cap T_{n}$, hence $U_{n}=T_{n}$. When $Z=R$, this is equivalent to one of $\mathrm{E}$. Cartan's theorems 
on the real forms of the simple Lie groups [3, p. 286].

20. We end by mentioning some relations between our results and the properties of the commutator subgroup $C$ of a sfield $K$ with involution.

THEOREM 5. Let $K$ be a sfield of characteristic $\neq 2$, of finite rank over its center $Z$, and let $J$ be an involution in $K$ leaving invariant the elements of $Z$. Then, for every $\xi \in K^{*}, \xi$ and $\xi^{J}$ are in the same class modulo the commutator subgroup $C$ of $K^{*}$.

Let $m^{2}$ be the rank of $K$ over its center, and let us suppose first that the set $S$ of symmetric elements in $K$ has dimension $m(m+1) / 2$ over $Z$. Then we have seen in $\$ 14$ that every element $\xi \in K^{*}$ can be written $\xi=\alpha \beta$, where $\alpha$ and $\beta$ are in $S$; accordingly $\xi^{J}=\beta^{J} \alpha^{J}=\beta \alpha$, hence $\xi^{J} \xi^{-1}=\beta \alpha \beta^{-1} \alpha^{-1}$, which proves our contention in that case. If on the contrary $S$ has dimension $m(m-1) / 2$ over $Z$, and $p$ is a skew-symmetric element of $K$, then $\xi \rightarrow \xi^{T}$ $=p^{-1} \xi^{J} p$ is an involution in $K$ for which the symmetric elements form a space of dimension $m(m+1) / 2$ over $Z(\S 3)$; therefore $\xi$ and $\xi^{T}$ are in the same class modulo $C$, and the same is true for $\xi$ and $\xi^{J}$, since $\xi$ and $p^{-1} \xi p$ are in the same class modulo $C$.

The situation is reversed when $K$ is a sfield of the second kind:

THEOREM 6. Let $K$ be a sfield of finite rank over its center $Z$, and let $J$ be an involution in $K$ which does not leave invariant every element of $Z$. Then there exist elements $\xi$ in $K^{*}$ such that $\xi$ and $\xi^{J}$ are not in the same class modulo $C$.

The theorem being obvious when $K$ is commutative, we can suppose that $K$ is not commutative, hence that $Z$ is an infinite field. The theorem will be proved if we exhibit a homomorphism $\phi$ of $K^{*}$ onto an abelian group, such that $\phi\left(\xi^{J}\right) \neq \phi(\xi)$ for some $\xi \in K^{*}$. Let $N(\xi)$ be the norm of an element $\xi$ in the regular representation of $K$ (considered as an algebra over its center $Z$ ); $\xi \rightarrow N(\xi)$ is then a homomorphism of $K^{*}$ into $Z^{*}$. If $r=m^{2}$ is the rank of $K$ over $Z$, we have $N(\xi)=\xi^{r}$ for every $\xi \in Z^{*}$; we have only therefore to verify that if the element $\omega \in Z$ constitutes with the identity a basis of $Z$ over the subfield $Z_{0}$ of $J$-invariant elements, then the elements $(x+y \omega)^{r}$ and $\left(x+y \omega^{J}\right)^{r}$ cannot be identical for all values of $x$ and $y$ in $Z_{0}$. But as $\omega^{J} \neq \omega$, this follows at once from the fact that $Z_{0}$ is an infinite field.

Theorem 6 has as a consequence that when $K$ is a sfield of the second kind, the groups $U_{n}$ and $T_{n}$ (for $\left.\nu \geqq 1\right)$ are always distinct. To prove this, we have only to verify that the determinant [4] of some unitary matrix is not the identity element in $K^{*} / C$; but this is obvious for the matrix

$$
\left(\begin{array}{cc}
\alpha & 0 \\
0 & \left(\alpha^{-1}\right)^{J}
\end{array}\right)
$$

if $\alpha$ and $\alpha^{J}$ are not in the same class modulo $C$. 


\section{BIBLIOGRAPHY}

1. A. A. Albert, Involutorial simple algebras and real Riemann matrices, Ann. of Math. vol. 36 (1935) pp. 886-964.

2. E. Artin and G. Whaples, The theory of simple rings, Amer. J. Math. vol. 65 (1943) pp. 87-107.

3. E. Cartan, Les groupes réels simples, finis et continus, Ann. Ecole Norm. (3) vol. 31 (1914) pp. 263-355.

4. J. Dieudonné, Les déterminants sur un corps non commutatif, Bull. Soc. Math. France vol. 71 (1943) pp. 27-45.

5. - Sur les groupes classiques, Actualités Scientifiques et Industrielles, no. 1040, Paris, Hermann, 1948.

6. I. Kaplansky, Forms in infinite-dimensional spaces, Anais da Academia Brasileira de Ciencias vol. 22 (1950) pp. 1-17.

7. C. Rosati, Sulle matrici di Riemann, Rend. Circ. Mat. Palermo vol. 53 (1929) pp. 79134.

\section{UNIVERSITY OF NANCY,} NANCY, FrancE. 